\title{
Basic ionic liquid [bmIm] OH mediated Gewald reaction as green protocol for the synthesis of 2-aminothiophenes
}

\author{
Venkata Rao Kaki ${ }^{\text {, }}$ Raghuram Rao Akkinepalli ${ }^{\mathrm{b}}$, Prankishore Deb ${ }^{\mathrm{a}}$, Mallikarjuna Rao Pichika ${ }^{\mathrm{a}}$ \\ a Pharmaceutical Chemistry Division, School of Pharmacy, International Medical University \\ (IMU), No. 126, Jalan Jalil Perkasa 19, Bukit Jalil, 57000 Kuala Lumpur, Malaysia. b \\ Pharmaceutical Chemistry Division, University College of Pharmaceutical Sciences, Kakatiya \\ University, Warangal, A. P. India - 506009. kvenkatrao80@gmail.com, \\ VenkataRao@imu.edu.my
}

\section{Experimental protocols}

All the reagents used were as purchased without purification. Solvents used for reactions were dried according to standard methods. Melting points were recorded in open capillaries on Casiaa Siamea (VMP-AM) melting point apparatus and are uncorrected. IR spectra were recorded on a Perkin Elmer FT-IR 240-C spectrophotometer using KBr discs. ${ }^{1} \mathrm{H}$ NMR and ${ }^{13} \mathrm{C}$ NMR spectra were recorded on Bruker Avance II (400 MHz and $100 \mathrm{MHz}$ respectively) spectrometer in DMSO- $d_{6}$ or $\mathrm{CDCl}_{3}$ using TMS as internal standard. Mass spectra (ESI) were recorded on Waters Micromass Q-TOF Micro. All the reactions were monitored by thin layer chromatography (TLC) on precoated silica gel 60 F254 (mesh) (E. Merck, Mumbai) and spots were visualized under UV light $(254 \mathrm{~nm})$.

\section{Experimental Data}

2-amino-4,5-dimethylthiophene-3-carbonitrile (2a)

White solid; Mp $140{ }^{\circ} \mathrm{C}$ (lit. ${ }^{[22]} 89-90{ }^{\circ} \mathrm{C}$ ); IR (KBr): 3445, 3328, 2196, $1521 \mathrm{~cm}^{-1}$; ${ }^{1} \mathrm{H}$ NMR $\left(400 \mathrm{MHz}, \mathrm{CDCl}_{3}\right): \delta=4.7(\mathrm{~s}, 2 \mathrm{H}), 2.2(\mathrm{~s}, 3 \mathrm{H}), 2.28(\mathrm{~s}, 3 \mathrm{H})$. 
Ethyl 2-amino-4,5-dimethylthiophene-3-carboxylate (2b)

Brown color solid; $\mathrm{Mp} 90^{\circ} \mathrm{C}$ (lit. ${ }^{[22]} 89-90{ }^{\circ} \mathrm{C}$ ); IR (KBr): 3345, 3318, $1624 \mathrm{~cm}^{-1} ;{ }^{1} \mathrm{H}$ NMR (400 $\left.\mathrm{MHz}, \mathrm{CDCl}_{3}\right): \delta=5.17(\mathrm{~s}, 2 \mathrm{H}), 4.25(\mathrm{q}, J=6.7 \mathrm{~Hz}, 2 \mathrm{H}), 2.2(\mathrm{~s}, 3 \mathrm{H}), 2.28(\mathrm{~s}, 3 \mathrm{H}), 1.33(\mathrm{t}, J=6.7$ $\mathrm{Hz}, 3 \mathrm{H})$.

2-amino-5,6-dihydro-4H-cyclopenta[b]thiophene-3-carbonitrile (2c)

Brown color solid; Mp $161{ }^{\circ} \mathrm{C}$; IR (KBr): 3414, 3294, 2184, $1287 \mathrm{~cm}^{-1}$; ${ }^{1} \mathrm{H}$ NMR (400 MHz, $\left.\mathrm{CDCl}_{3}\right): \delta=4.73(\mathrm{~s}, 2 \mathrm{H}), 2.80(\mathrm{~m}, 2 \mathrm{H}), 2.74(\mathrm{~m}, 2 \mathrm{H}), 2.36(\mathrm{~m}, 2 \mathrm{H}) ; \mathrm{MS}(\mathrm{ESI}): \mathrm{m} / z=212.2$ $(\mathrm{M}+\mathrm{H})^{+}$.

Ethyl 2-Amino-5,6-dihydro-4H-cyclopenta[b]thiophene-3-carboxylate (2d)

Brown color solid; Mp $90{ }^{\circ} \mathrm{C}$ (lit. ${ }^{[23]} 89-90{ }^{\circ} \mathrm{C}$ ); IR (KBr): 3414, 3294, 1624, $1287 \mathrm{~cm}^{-1} ;{ }^{1} \mathrm{H}$ $\operatorname{NMR}\left(400 \mathrm{MHz}, \mathrm{CDCl}_{3}\right): \delta=5.83(\mathrm{~s}, 2 \mathrm{H}), 4.25(\mathrm{q}, J=7.1 \mathrm{~Hz}, 2 \mathrm{H}), 2.85-2.80(\mathrm{~m}, 2 \mathrm{H}), 2.74(\mathrm{~m}$, 2H), $2.36(\mathrm{~m}, 2 \mathrm{H}), 1.33(\mathrm{t}, J=7.1 \mathrm{~Hz}, 3 \mathrm{H}) ;{ }^{13} \mathrm{C} \mathrm{NMR}\left(100 \mathrm{MHz}, \mathrm{CDCl}_{3}\right): \delta=166.2,165.8$, 142.7, 121.4, 103.0, 59.4, 30.8, 28.9, 27.2, 14.4.; MS (ESI): $m / z=212.2(\mathrm{M}+\mathrm{H})^{+}$.

2-Amino-4,5,6,7-tetrahydrobenzo[b]thiophene-3-carbonitrile (2e)

White solid; Mp $145^{\circ} \mathrm{C}$ (lit. ${ }^{[23]} 146-147{ }^{\circ} \mathrm{C}$ ); IR (KBr): 3445, 3328, 2196, $1521 \mathrm{~cm}^{-1}$; ${ }^{1} \mathrm{H}$ NMR $\left(400 \mathrm{MHz}, \mathrm{CDCl}_{3}\right): \delta=4.71(\mathrm{~s}, 2 \mathrm{H}), 2.48(\mathrm{~m}, 4 \mathrm{H}), 1.79(\mathrm{~m}, 4 \mathrm{H}) ;{ }^{13} \mathrm{C}$ NMR $\left(100 \mathrm{MHz}, \mathrm{CDCl}_{3}\right)$ : $\delta=160.1,132.3,120.6,115.6,88.5,24.5,24.1,23.4,22.1$; MS (ESI): $m / z=178.3(\mathrm{M}+\mathrm{H})^{+}$.

Ethyl 2-Amino-4,5,6,7-tetrahydrobenzo[b]thiophene-3-carboxylate (2f)

White solid; Mp $113{ }^{\circ} \mathrm{C}$ (lit. ${ }^{[23]} 115{ }^{\circ} \mathrm{C}$ ); IR (KBr) 3405, 3299, 1647, $1274 \mathrm{~cm}^{-1} ;{ }^{1} \mathrm{H}$ NMR (400 $\left.\mathrm{MHz}, \mathrm{CDCl}_{3}\right): \delta=6.01(\mathrm{~s}, 2 \mathrm{H}), 4.25(\mathrm{q}, J=7.2 \mathrm{~Hz}, 2 \mathrm{H}), 2.71(\mathrm{~m}, 2 \mathrm{H}), 2.48(\mathrm{~m}, 2 \mathrm{H}), 1.75(\mathrm{~m}$, $4 \mathrm{H}), 1.33(\mathrm{t}, \mathrm{J}=7.2 \mathrm{~Hz}, 3 \mathrm{H}) ;{ }^{13} \mathrm{C} \mathrm{NMR}\left(100 \mathrm{MHz}, \mathrm{CDCl}_{3}\right): \delta=166.6,161.9,132.4,117.5$, 105.5, 59.4, 27.0, 24.5, 23.3, 22.9, 14.5. MS (ESI): $\mathrm{m} / z=225.4(\mathrm{M}+\mathrm{H})^{+}$.

2-Amino-5,6,7,8-tetrahydro-4H-cyclohepta[b]thiophene-3-carbonitrile (2g) 
White solid; Mp $125^{\circ} \mathrm{C}$ (lit. ${ }^{[24]} 126{ }^{\circ} \mathrm{C}$ ); IR (KBr): 3443, 3310, $2203 \mathrm{~cm}^{-1} ;{ }^{1} \mathrm{H}$ NMR (400 MHz, $\left.\mathrm{CDCl}_{3}\right): \delta=4.26(\mathrm{~s}, 2 \mathrm{H}), 2.65(\mathrm{~m}, 4 \mathrm{H}), 1.83(\mathrm{~m}, 2 \mathrm{H}), 1.67(\mathrm{~m}, 4 \mathrm{H}) .{ }^{13} \mathrm{C}$ NMR $(100 \mathrm{MHz}$, $\left.\mathrm{CDCl}_{3}\right): \delta=157.7,136.9,123.9,115.9,92.0,31.9,29.4,29.2,28.1,27.2 . ;$ MS (ESI): $\mathrm{m} / \mathrm{z}=$ $192.3(\mathrm{M}+\mathrm{H})^{+}$.

Ethyl 2-Amino-5,6,7,8-tetrahydro-4H-cyclohepta[b]thiophene-3-carboxylate (2h)

White solid; Mp $87^{\circ} \mathrm{C}$ (lit. ${ }^{[24]} 89^{\circ} \mathrm{C}$ ); IR (KBr): 3398, 3301, 1651, $1277 \mathrm{~cm}^{-1}$.; ${ }^{1} \mathrm{H}$ NMR (400 $\left.\mathrm{MHz}, \mathrm{CDCl}_{3}\right): \delta=5.74(\mathrm{~s}, 2 \mathrm{H}), 4.28(\mathrm{q}, J=7.2 \mathrm{~Hz}, 2 \mathrm{H}), 3.00(\mathrm{~m}, 2 \mathrm{H}), 2.60(\mathrm{~m}, 2 \mathrm{H}), 1.82(\mathrm{~m}$, $2 \mathrm{H}), 1.68(\mathrm{~m}, 4 \mathrm{H}), 1.35(\mathrm{t}, J=7.2 \mathrm{~Hz}, 3 \mathrm{H}) .{ }^{13} \mathrm{C} \mathrm{NMR}\left(100 \mathrm{MHz}, \mathrm{CDCl}_{3}\right): \delta=166.0,159.7$, 137.9, 121.4, 107.6, 59.6, 32.2, 28.7, 28.6, 27.8, 27.0, 14.5.; MS (ESI): $\mathrm{m} / z=239.4(\mathrm{M}+\mathrm{H})^{+}$.

2-amino-6-benzyl-4,5,6,7-tetrahydrothieno[2,3-c]pyridine-3-carbonitrile (2i)

Red color solid; Mp 89-90 ${ }^{\circ} \mathrm{C} ;{ }^{1} \mathrm{H}$ NMR (400 MHz, $\left.\mathrm{CDCl}_{3}\right): \delta=7.31$ (m, 5H), $4.68(\mathrm{~s}, 2 \mathrm{H}), 3.69$ (s, 2H), $3.40(\mathrm{~s}, 2 \mathrm{H}), 2.80(\mathrm{t}, J=6.0 \mathrm{~Hz}, 2 \mathrm{H}), 2.63(\mathrm{t}, J=6.0 \mathrm{~Hz}, 2 \mathrm{H}) \cdot{ }^{13} \mathrm{C}$ NMR $(100 \mathrm{MHz}$, $\left.\mathrm{CDCl}_{3}\right): \delta=160.5,137.8,130.9,129.9,128.4,127.3,117.8,115.1,88.2,61.7,50.7,49.5,24.6$. MS (ESI): $m / z=270.1(\mathrm{M}+\mathrm{H})^{+}$.

Ethyl 2-amino-6-benzyl-4,5,6,7-tetrahydrothieno[2,3-c]pyridine-3-carboxylate (2l)

Brick red color solid; ${ }^{1} \mathrm{H}$ NMR (400 MHz, $\left.\mathrm{CDCl}_{3}\right): \delta=7.31(\mathrm{~m}, 5 \mathrm{H}), 5.7$ (s, 2H), 4.28 (q, J = 7.2 $\mathrm{Hz}, 2 \mathrm{H}), 3.69(\mathrm{~s}, 2 \mathrm{H}), 3.40(\mathrm{~s}, 2 \mathrm{H}), 2.81(\mathrm{t}, J=6.0 \mathrm{~Hz}, 2 \mathrm{H}), 2.63(\mathrm{t}, J=6.0 \mathrm{~Hz}, 2 \mathrm{H}), 1.35(\mathrm{t}, J=$ $7.2 \mathrm{~Hz}, 3 \mathrm{H}) ; \mathrm{MS}(\mathrm{ESI}): \mathrm{m} / \mathrm{z}=316.9(\mathrm{M}+\mathrm{H})^{+}$.

2-Amino-4-phenyl-3-thiophenecarbonitrile (5a)

White solid; Mp 100-102 ${ }^{\circ} \mathrm{C}$ (lit. ${ }^{[25]} 100-102{ }^{\circ} \mathrm{C}$ ); ${ }^{1} \mathrm{H}$ NMR (400 MHz, $\mathrm{CDCl}_{3}$ ): $\delta=7.48-7.56$ $(\mathrm{m}, 2 \mathrm{H}), 7.38(\mathrm{~m}, 2 \mathrm{H}), 7.30(\mathrm{~m}, 1 \mathrm{H}), 7.23(\mathrm{~s}, 2 \mathrm{H}), 6.51(\mathrm{~s}, 1 \mathrm{H}) ;{ }^{13} \mathrm{C} \mathrm{NMR}\left(100 \mathrm{MHz}, \mathrm{CDCl}_{3}\right): \delta$ $=165.7,137.9,133.9,128.1,127.3,126.4,116.2,104.7,82.9 . ; \mathrm{MS}(\mathrm{ESI}): m / z=201.0(\mathrm{M}+\mathrm{H})^{+}$. 2-Amino-4-(4-methoxyphenyl)-3-thiophenecarbonitrile (5b) 
White solid; Mp 164-166 ${ }^{\circ} \mathrm{C}$ (lit. $\left.{ }^{[26]} 166{ }^{\circ} \mathrm{C}\right) .{ }^{1} \mathrm{H}$ NMR $\left(400 \mathrm{MHz}, \mathrm{CDCl}_{3}\right): \delta=7.45$ (d, $J=8.9$ $\mathrm{Hz}, 2 \mathrm{H}), 7.18$ (s, 2H), 6.97 (d, J = 8.9 Hz, 2H), 6.39 (s, 1H), 3.77 (s, 3H); ${ }^{13} \mathrm{C}$ NMR (100 MHz, $\left.\mathrm{CDCl}_{3}\right): \delta=165.6,158.3,137.6,127.6,126.5,116.3,113.6,103.3,83.1,55.1 . ; \mathrm{MS}(\mathrm{ESI}): \mathrm{m} / \mathrm{z}=$ $231(\mathrm{M}+\mathrm{H})^{+}$.

2-Amino-4,5-diphenyl-3-thiophenecarbonitrile (5c)

Brown color solid; Mp 203-205 ${ }^{\circ} \mathrm{C}$ (lit. $\left.{ }^{[26]} 205{ }^{\circ} \mathrm{C}\right) ;{ }^{1} \mathrm{H}$ NMR $\left(400 \mathrm{MHz}, \mathrm{CDCl}_{3}\right): \delta=7.50(\mathrm{~m}$, 3H), $7.42(\mathrm{~m}, 2 \mathrm{H}), 7.28(\mathrm{~m}, 3 \mathrm{H}), 7.26(\mathrm{~m}, 2 \mathrm{H}) .{ }^{13} \mathrm{C} \mathrm{NMR}\left(100 \mathrm{MHz}, \mathrm{CDCl}_{3}\right): \delta=163.3,134.1$, 133.7, 132.4, 128.8, 128.1, 127.6, 127.4, 126.4, 118.8, 115.7, 86.6.; MS (ESI): $\mathrm{m} / \mathrm{z}=277.2$ $(\mathrm{M}+\mathrm{H})^{+}$.

2-Amino-4-(4-bromophenyl)thiophene-3-carbonitrile (5d)

Yellow solid; Mp 190-191 ${ }^{\circ} \mathrm{C}$ (lit. ${ }^{[25]}$ 190-192 ${ }^{\circ} \mathrm{C}$ ); ${ }^{1} \mathrm{H}$ NMR (400 MHz, DMSO-do): $\delta 7.61$ (d, $J$ $=8.0 \mathrm{~Hz}, 2 \mathrm{H}), 7.48(\mathrm{~d}, J=8.4 \mathrm{~Hz}, 2 \mathrm{H}), 7.29(\mathrm{~s}, 2 \mathrm{H}), 6.56(\mathrm{~s}, 1 \mathrm{H}), 4.87(\mathrm{~s}, 2 \mathrm{H}),{ }^{13} \mathrm{C}$ NMR $(100$ MHz, DMSO-d $\left.{ }_{6}\right): \delta 167.0,137.5,134.0,132.0,129.3,121.5,116.9,106.1,83.2$.

Diethyl 5-amino-3-methylthiophene-2,4-dicarboxylate (5g)

Brown color solid; Mp. $108-109{ }^{\circ} \mathrm{C}$ (lit. ${ }^{17} 106{ }^{\circ} \mathrm{C}$ ); IR (KBr): 3408, 3294, 1682, 1660, 1587, 1529, $1232 \mathrm{~cm}^{-1} ;{ }^{1} \mathrm{H}$ NMR $\left(400 \mathrm{MHz}, \mathrm{CDCl}_{3}\right): \delta=1.33(\mathrm{t}, J=7.1 \mathrm{~Hz}, 3 \mathrm{H}), 1.37(\mathrm{t}, J=7.1 \mathrm{~Hz}$, 3H), $2.70(\mathrm{~s}, 3 \mathrm{H}), 4.26(\mathrm{q}, J=7.1 \mathrm{~Hz}, 2 \mathrm{H}), 4.31(\mathrm{q}, J=7.1 \mathrm{~Hz}, 2 \mathrm{H}), 6.60(\mathrm{~s}, 2 \mathrm{H}) \cdot ;{ }^{13} \mathrm{C}$ NMR $(100$ $\left.\mathrm{MHz}, \mathrm{CDCl}_{3}\right): \delta=14.5,14.6,16.3,60.2,60.6,108.6,108.7,148.2,163.1,166.3,166.4 . ; \mathrm{MS}$ (ESI): $m / z=257.0(\mathrm{M}+\mathrm{H})^{+}$.

2-amino-4-methoxythiophene-3-carbonitrile (8a)

Black colour solid; Mp. $78-79{ }^{\circ} \mathrm{C} ;{ }^{1} \mathrm{H}$ NMR $\left(400 \mathrm{MHz}, \mathrm{CDCl}_{3}\right): \delta=5.21(\mathrm{~s}, 1 \mathrm{H}), 4.89(\mathrm{~s}, 2 \mathrm{H}$, $\left.\mathrm{NH}_{2}\right), 3.79$ (s, $\left.3 \mathrm{H}, \mathrm{OCH}_{3}\right) .{ }^{13} \mathrm{C} \mathrm{NMR}\left(100 \mathrm{MHz}, \mathrm{CDCl}_{3}\right): \delta=160.8,154.1,113.9,82.6,79.9$, 57.0. MS (ESI): $m / z=155.02(\mathrm{M}+\mathrm{H})^{+}$. 
2-amino-4-ethoxythiophene-3-carbonitrile (8b)

Brown colour solid; Mp $134{ }^{\circ} \mathrm{C}$; IR (KBr): 3439, 3320, 3213, 2972, 2199, 1631, $1557 \mathrm{~cm}^{-1}$.; ${ }^{1} \mathrm{HNMR}\left(400 \mathrm{MHz}, \mathrm{CDCl}_{3}\right): \delta=5.18(\mathrm{~s}, 1 \mathrm{H}), 4.79\left(\mathrm{~s}, 2 \mathrm{H}, \mathrm{NH}_{2}\right), 4.01(\mathrm{q}, J=6.8 \mathrm{~Hz}, 2 \mathrm{H}), 1.40(\mathrm{t}$, $J=6.8 \mathrm{~Hz}, 3 \mathrm{H}) .{ }^{13} \mathrm{C} \mathrm{NMR}\left(100 \mathrm{MHz}, \mathrm{CDCl}_{3}\right): \delta=160.4,154.2,113.9,82.6,78.9,59.0,15.1$; $\operatorname{MS}\left(\mathrm{ESI}^{+}\right): m / z=169.1(\mathrm{M}+\mathrm{H})$.

2-amino-4-propoxythiophene-3-carbonitrile (8c)

Cream colour solid; $\mathrm{Mp} 220^{\circ} \mathrm{C}$; IR (KBr): 3436, 3320, 3215, 2934, 2877, 2205, 1632, $1559 \mathrm{~cm}^{-}$

${ }^{1} ;{ }^{1} \mathrm{H}$ NMR (400 MHz, $\left.\mathrm{CDCl}_{3}\right): \delta 5.18(\mathrm{~s}, 1 \mathrm{H}), 4.77\left(\mathrm{~s}, 2 \mathrm{H}, \mathrm{NH}_{2}\right), 3.85(\mathrm{t}, J=6.8 \mathrm{~Hz}, 2 \mathrm{H}), 1.82$ $(\mathrm{m}, 2 \mathrm{H}), 1.01(\mathrm{t}, J=6.8 \mathrm{~Hz}, 3 \mathrm{H}) .{ }^{13} \mathrm{C} \mathrm{NMR}\left(100 \mathrm{MHz}, \mathrm{CDCl}_{3}\right): \delta 160.4,153.6,113.9,83.1$, 78.9, 62.1, 22.1, 17.5.; $\mathrm{MS}\left(\mathrm{ESI}^{+}\right): m / z 182.9(\mathrm{M}+\mathrm{H})^{+}$

2-amino-4-butoxythiophene-3-carbonitrile (8d)

White solid; Mp $84{ }^{\circ} \mathrm{C}$; IR (KBr): 3438, 3339, 3221, 2951, 2867, 2198, 1620, $1555 \mathrm{~cm}^{-1} ;{ }^{1} \mathrm{H}$ NMR (400 MHz, $\left.\mathrm{CDCl}_{3}\right): \delta 5.17(\mathrm{~s}, 1 \mathrm{H}), 4.88\left(\mathrm{~s}, 2 \mathrm{H}, \mathrm{NH}_{2}\right), 3.92(\mathrm{t}, J=6.4 \mathrm{~Hz}, 2 \mathrm{H}), 1.76(\mathrm{~m}$, 2H), $1.47(\mathrm{~m}, 2 \mathrm{H}), 0.97(\mathrm{t}, J=7.2 \mathrm{~Hz}, 2 \mathrm{H}) .{ }^{13} \mathrm{C} \mathrm{NMR}\left(100 \mathrm{MHz}, \mathrm{CDCl}_{3}\right): \delta 160.1,153.3,113.8$, 83.2, 80.3, 74.2, 28.0, 21.1, 12.1.; $\mathrm{MS}\left(\mathrm{ESI}^{+}\right): \mathrm{m} / z 197.0(\mathrm{M}+\mathrm{H})^{+}$.

2-amino-4-isobutoxythiophene-3-carbonitrile (8e)

Brown colour solid; Mp $104{ }^{\circ} \mathrm{C}$; IR (KBr): 3433, 3322, 3207, 2958, 2207, 1622, $1557 \mathrm{~cm}^{-1}$.; ${ }^{1} \mathrm{HNMR}\left(400 \mathrm{MHz}, \mathrm{CDCl}_{3}\right): \delta=5.16(\mathrm{~s}, 1 \mathrm{H}), 4.79\left(\mathrm{~s}, 2 \mathrm{H}, \mathrm{NH}_{2}\right), 3.67(\mathrm{~d}, J=6.4 \mathrm{~Hz}, 2 \mathrm{H}), 2.1(\mathrm{~m}$, $1 \mathrm{H}), 1.01(\mathrm{~d}, J=6.4 \mathrm{~Hz}, 6 \mathrm{H}) .{ }^{13} \mathrm{C} \mathrm{NMR}\left(100 \mathrm{MHz}, \mathrm{CDCl}_{3}\right): \delta=160.3,153.5,113.8,83.2,80.2$, 76.25, 28.0, 14.1.; $\mathrm{MS}\left(\mathrm{ESI}^{+}\right): \mathrm{m} / z=197.0(\mathrm{M}+\mathrm{H})^{+}$.

2-amino-4-(pentyloxy)thiophene-3-carbonitrile (8f)

White solid; Mp $78{ }^{\circ} \mathrm{C}$; IR (KBr): 3446, 3347, 3225, 2942, 2864, 2199, 1621, $1554 \mathrm{~cm}^{-1}$.; ${ }^{1} \mathrm{HNMR}\left(400 \mathrm{MHz}, \mathrm{CDCl}_{3}\right): \delta=5.17(\mathrm{~s}, 1 \mathrm{H}), 4.8\left(\mathrm{~s}, 2 \mathrm{H}, \mathrm{NH}_{2}\right), 3.91(\mathrm{t}, J=6.4 \mathrm{~Hz}, 2 \mathrm{H}), 1.80(\mathrm{~m}$, 
2H), $1.43(\mathrm{~m}, 4 \mathrm{H}), 0.94(\mathrm{t}, J=6.4 \mathrm{~Hz}, 3 \mathrm{H}) .{ }^{13} \mathrm{C} \mathrm{NMR}\left(100 \mathrm{MHz}, \mathrm{CDCl}_{3}\right): \delta=160.4,153.4$, 113.9, 83.2, 80.2, 70.1, 28.5, 28.0, 22.4, 13.9.; $\mathrm{MS}_{\left(\mathrm{ESI}^{+}\right): \mathrm{m} / z=211.1(\mathrm{M}+\mathrm{H})^{+} .}$

2-amino-4-(octyloxy)thiophene-3-carbonitrile (8g)

White solid; Mp $82{ }^{\circ} \mathrm{C}$; IR (KBr): 3445, 3320, 3213, 2924, 2852, 2205, 1626, $1558 \mathrm{~cm}^{-1}$.; ${ }^{1} \mathrm{HNMR}\left(400 \mathrm{MHz}, \mathrm{CDCl}_{3}\right): \delta=5.17(\mathrm{~s}, 1 \mathrm{H}), 4.83\left(\mathrm{~s}, 2 \mathrm{H}, \mathrm{NH}_{2}\right), 3.91(\mathrm{t}, J=6.4 \mathrm{~Hz}, 2 \mathrm{H}), 1.78(\mathrm{~m}$, $2 \mathrm{H}), 1.46(\mathrm{~m}, 2 \mathrm{H}), 1.32(\mathrm{~m}, 8 \mathrm{H}), 0.88(\mathrm{~d}, J=6.4 \mathrm{~Hz}, 3 \mathrm{H}) \cdot{ }^{13} \mathrm{C}$ NMR $\left(100 \mathrm{MHz}, \mathrm{CDCl}_{3}\right): \delta=$ 160.1, 153.4, 113.9, 83.2, 80.2, 64.1, 28.5, 28.0, 22.4, 19.8, 19.3, 19.2, 11.9.; MS $\left(\mathrm{ESI}^{+}\right) \mathrm{m} / \mathrm{z}=$ $253.1(\mathrm{M}+\mathrm{H})^{+}$

2-amino-4-(benzyloxy)thiophene-3-carbonitrile (8h)

Black colour solid; IR (KBr): 3311, 3172, 2927, 2211, 1646, $1513 \mathrm{~cm}^{-1}$;; ${ }^{1} \mathrm{HNMR}(400 \mathrm{MHz}$, $\left.\mathrm{CDCl}_{3}\right): \delta=7.36-7.25(\mathrm{~m}, 5 \mathrm{H}), 5.18(\mathrm{~s}, 1 \mathrm{H}), 4.60\left(\mathrm{~s}, 2 \mathrm{H}, \mathrm{NH}_{2}\right), 3.69(\mathrm{~s}, 2 \mathrm{H}) .{ }^{13} \mathrm{C} \mathrm{NMR}$ $\left(100 \mathrm{MHz}, \mathrm{CDCl}_{3}\right): \delta=163.0,153.1,143.4,129.2,124.1,121.6,113.9,83.2,80.2 .70 .1 . ; \mathrm{MS}$ $\left(\mathrm{ESI}^{+}\right) \mathrm{m} / z=231.0(\mathrm{M}+\mathrm{H})^{+}$

2-(1-morpholinoethylidene)malononitrile (7i)

White solid; Mp $92{ }^{\circ} \mathrm{C} ;{ }^{1} \mathrm{HNMR}(400 \mathrm{MHz}$, DMSO- $d 6): \delta=3.73(\mathrm{~m}, 8 \mathrm{H}), 2.2(\mathrm{~s}, 3 \mathrm{H}) .{ }^{13} \mathrm{C}$ NMR (100MHz, DMSO-d6), $\delta$ (ppm): 168.6, 118.5, 117.1, 65.6, 49.9, 48.9, 20.5.

2-amino-4-morpholinothiophene-3-carbonitrile (8i)

Brown colour solid; Mp $126{ }^{\circ} \mathrm{C} ;{ }^{1} \mathrm{H}$ NMR (400MHz, DMSO- $\left.d 6\right): \delta=6.96(\mathrm{~s}, 2 \mathrm{H}), 5.31(\mathrm{~s}, 1 \mathrm{H})$, $3.7(\mathrm{t}, J=4.8 \mathrm{~Hz}, 4 \mathrm{H}), 2.9(\mathrm{t}, J=4.8 \mathrm{~Hz}, 4 \mathrm{H}) .{ }^{13} \mathrm{C}$ NMR (100MHz, DMSO-d6): $\delta=164.8$, $148.5,115.9,86.2,79.9,65.8,50.9 ; \mathrm{MS}\left(\mathrm{ESI}^{+}\right) \mathrm{m} / \mathrm{z}=210.0(\mathrm{M}+\mathrm{H})^{+}$. 

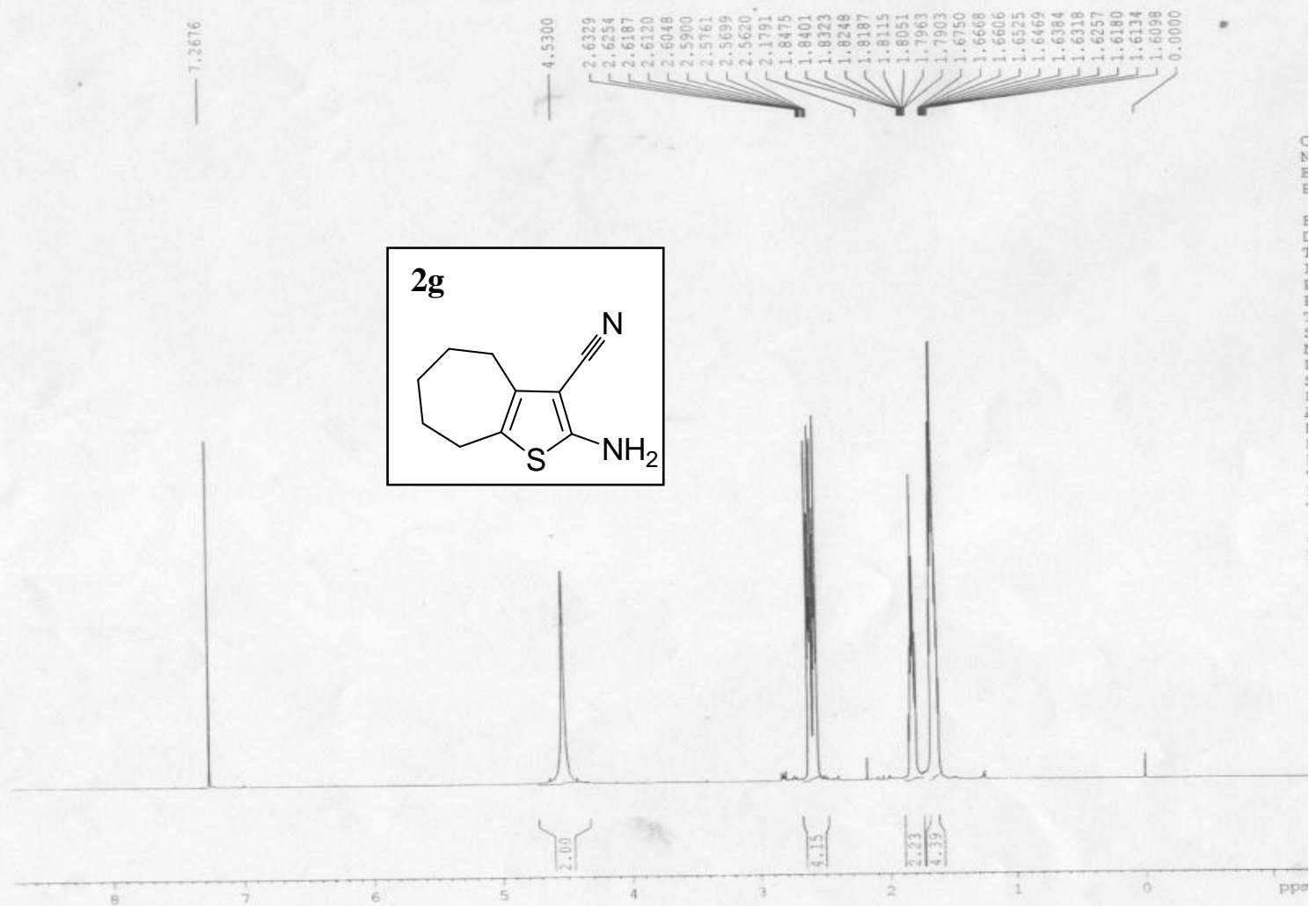

HEPT
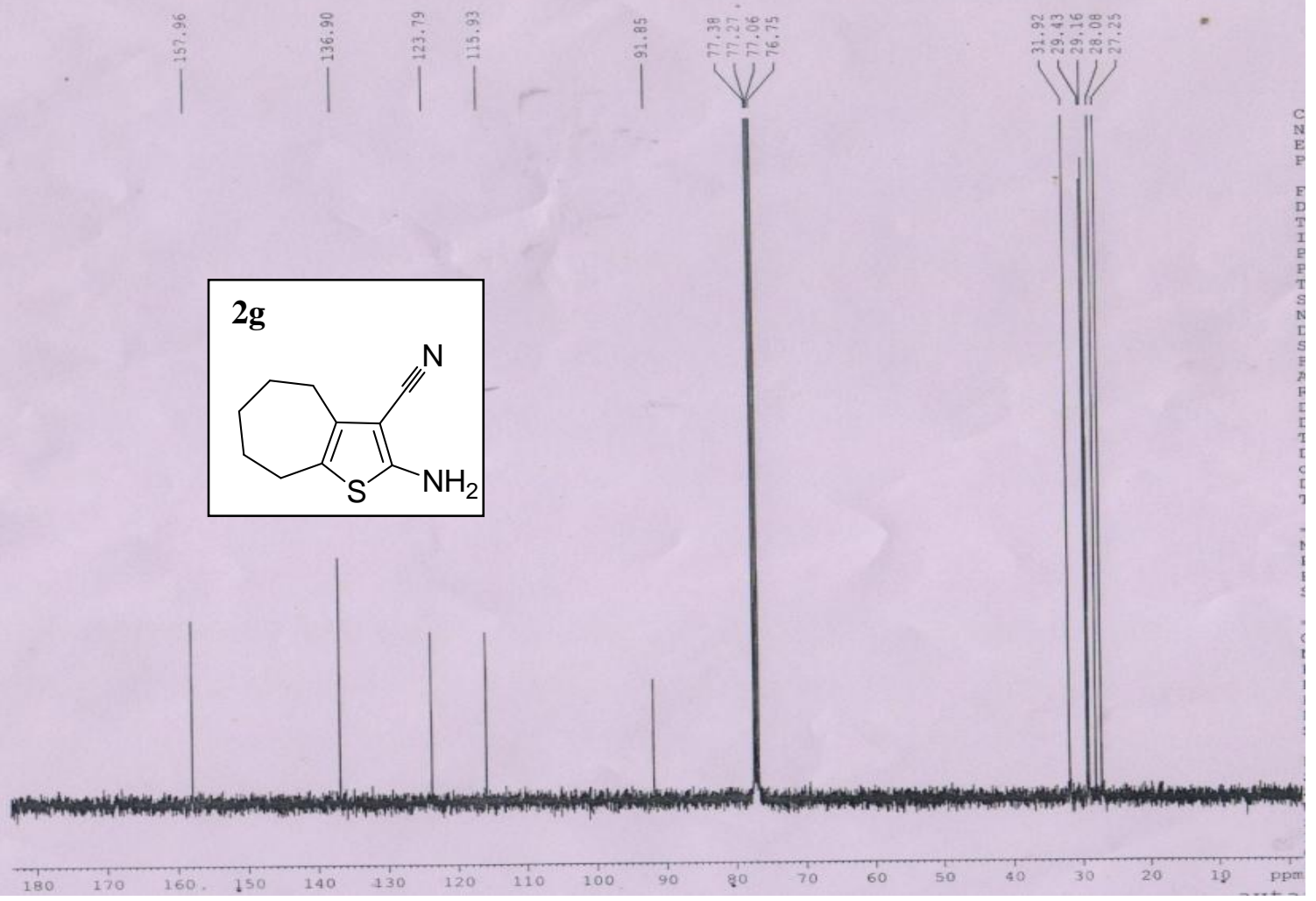


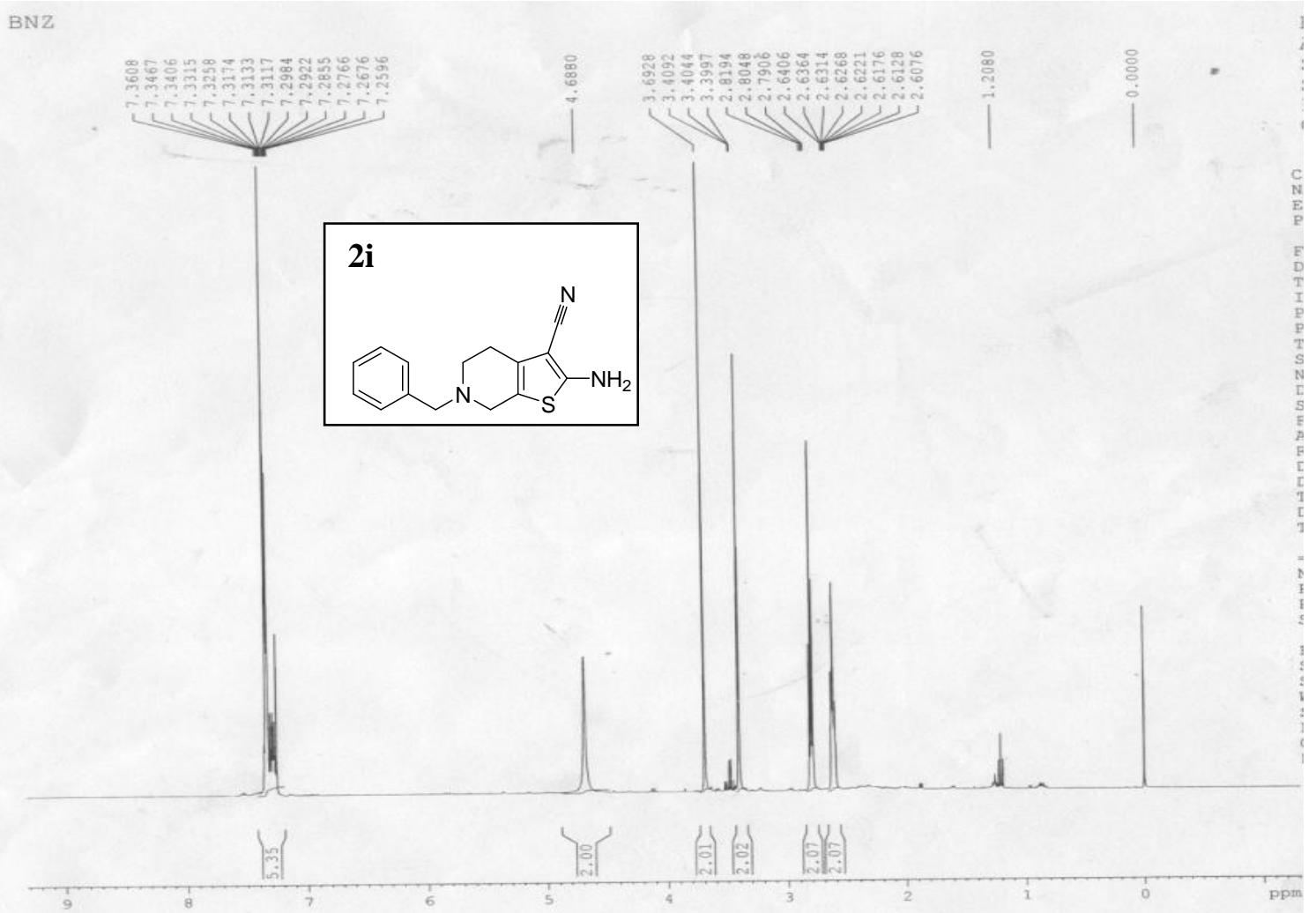

BNZ
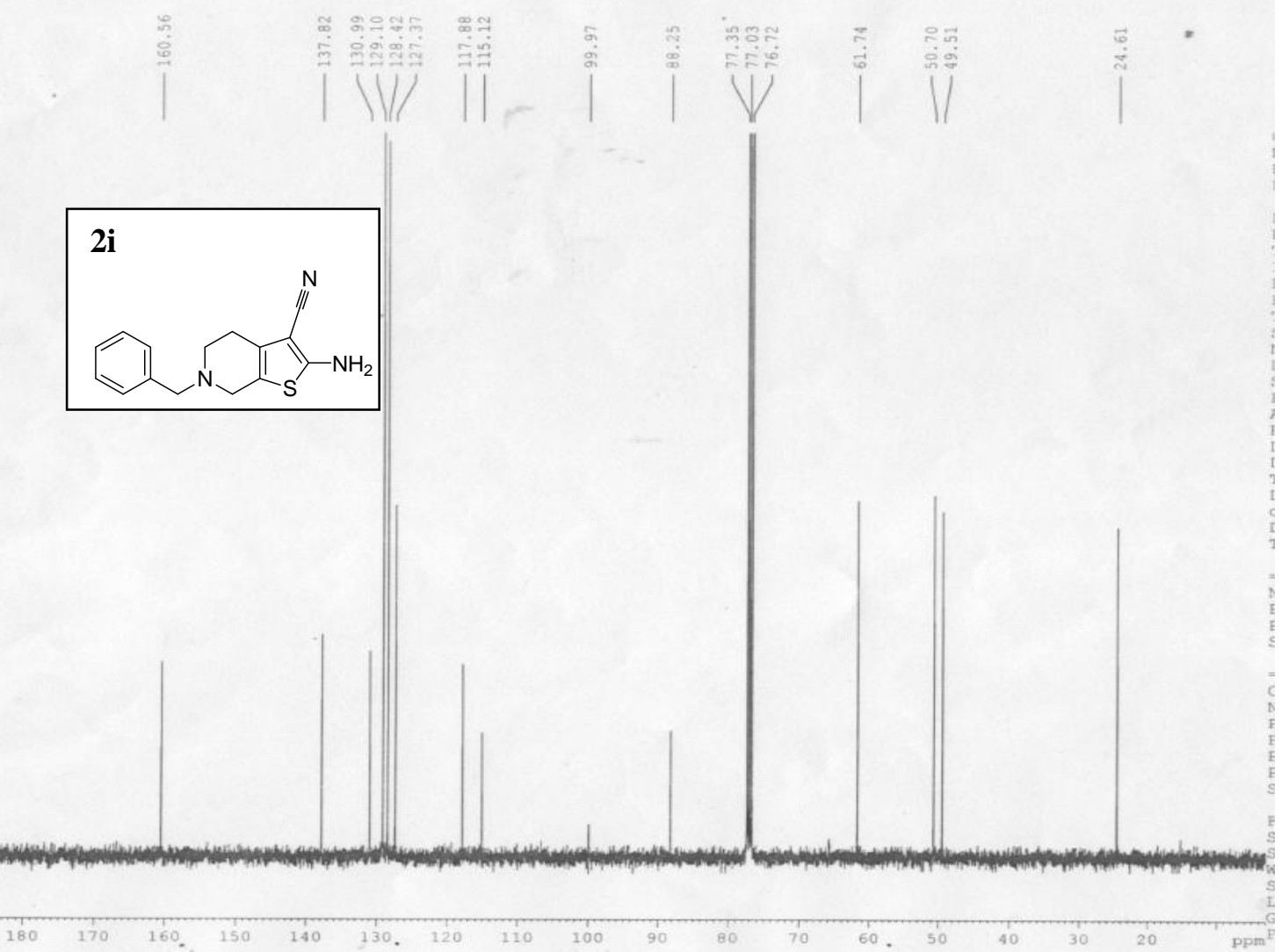

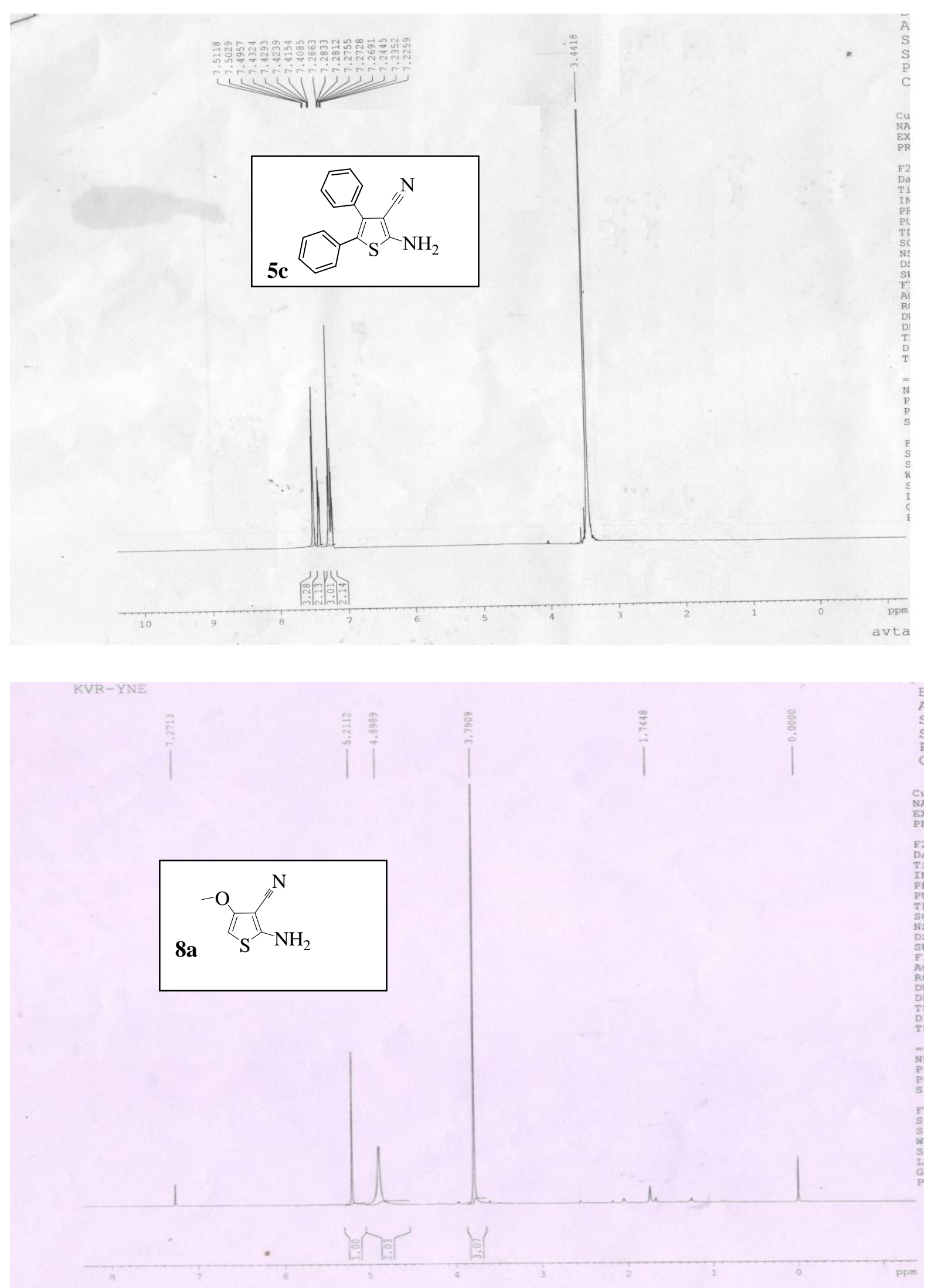

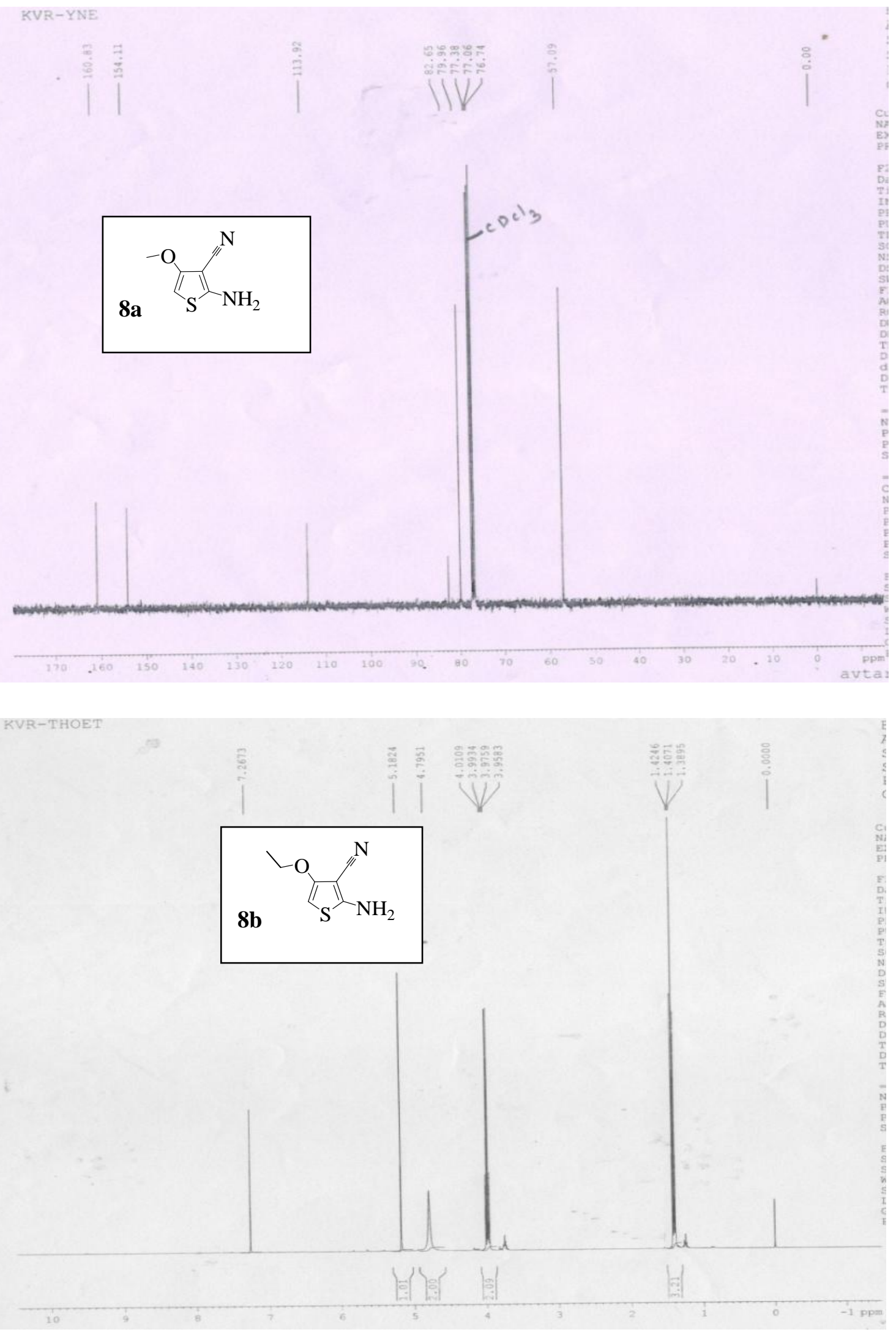

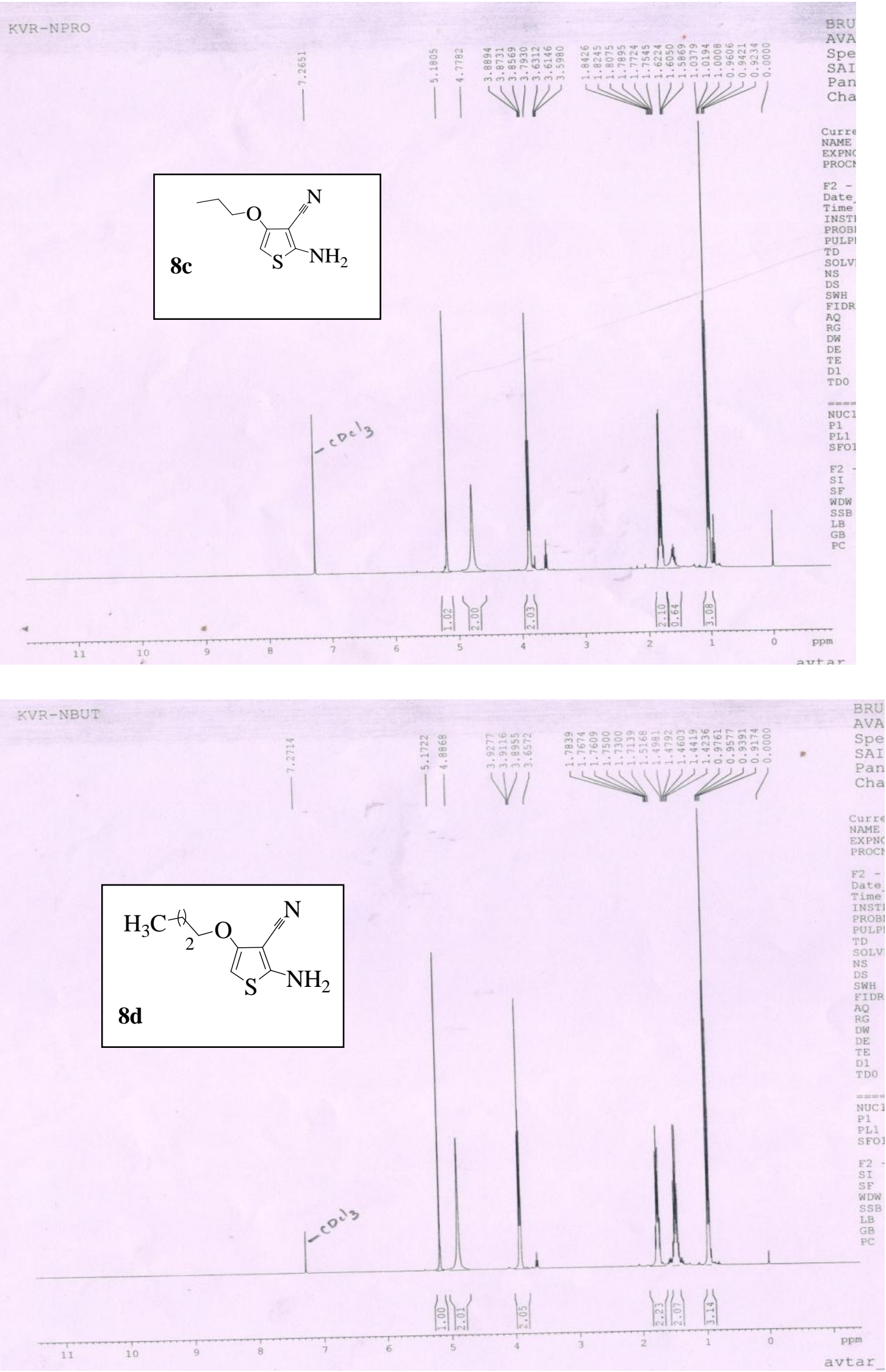

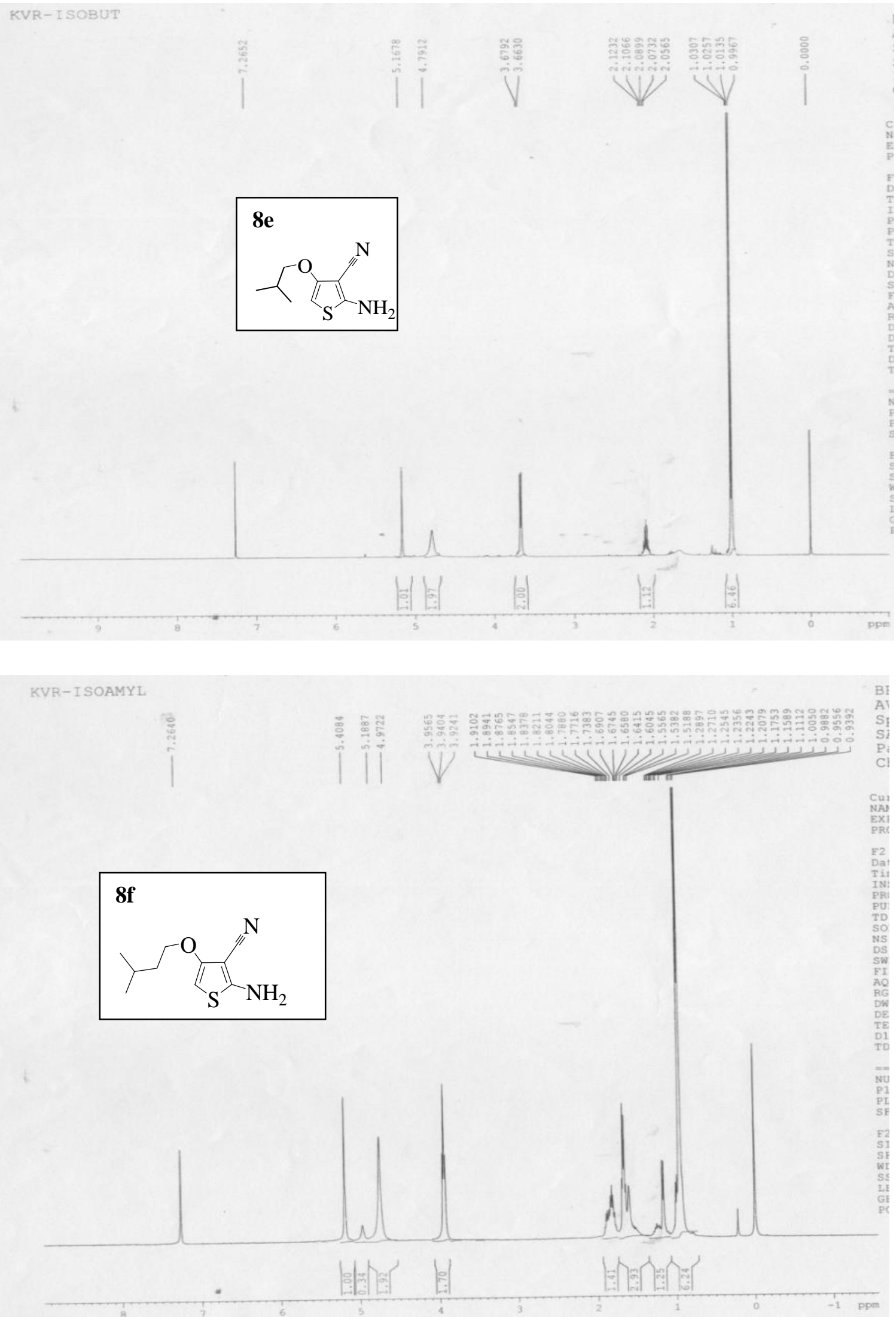

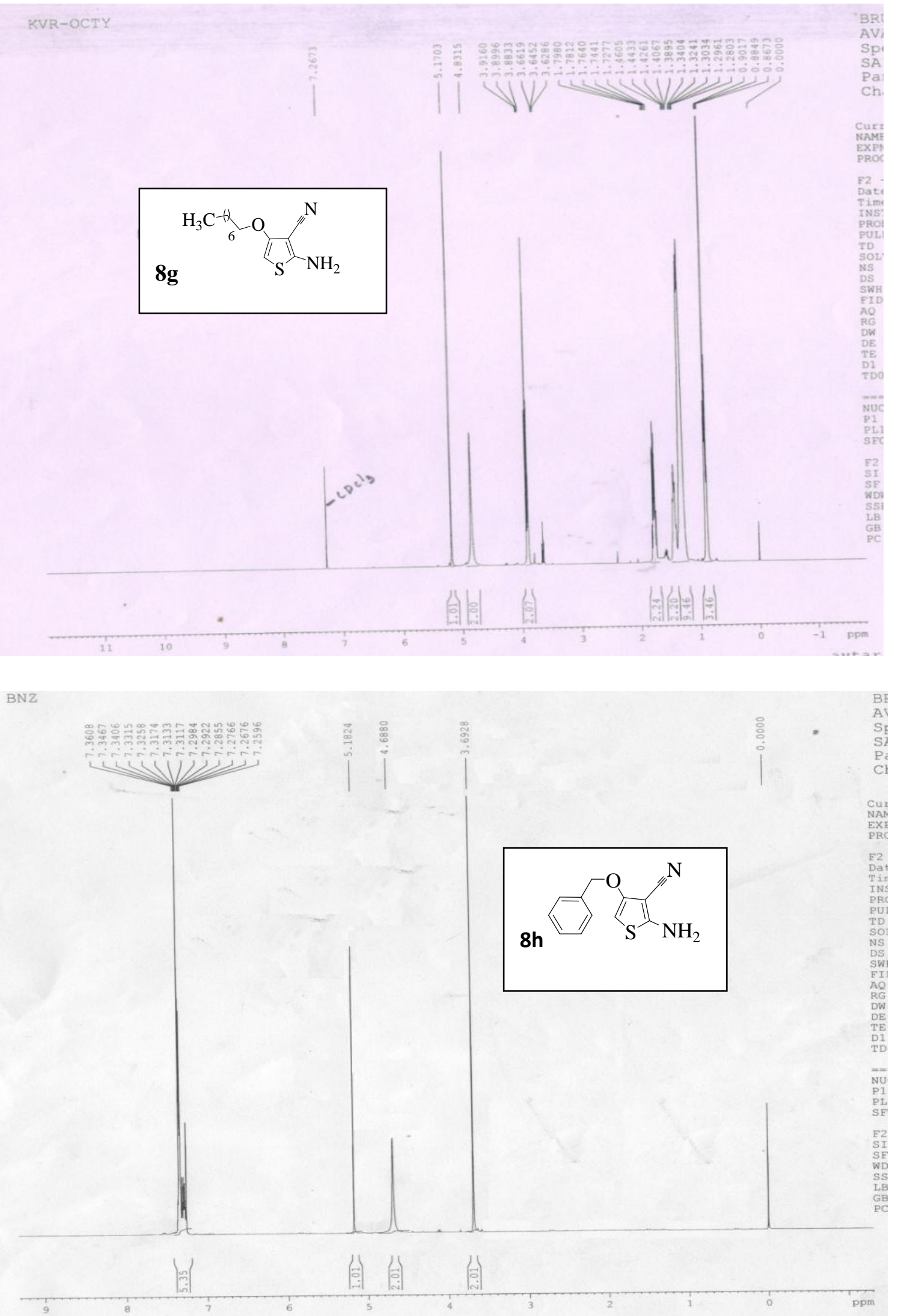

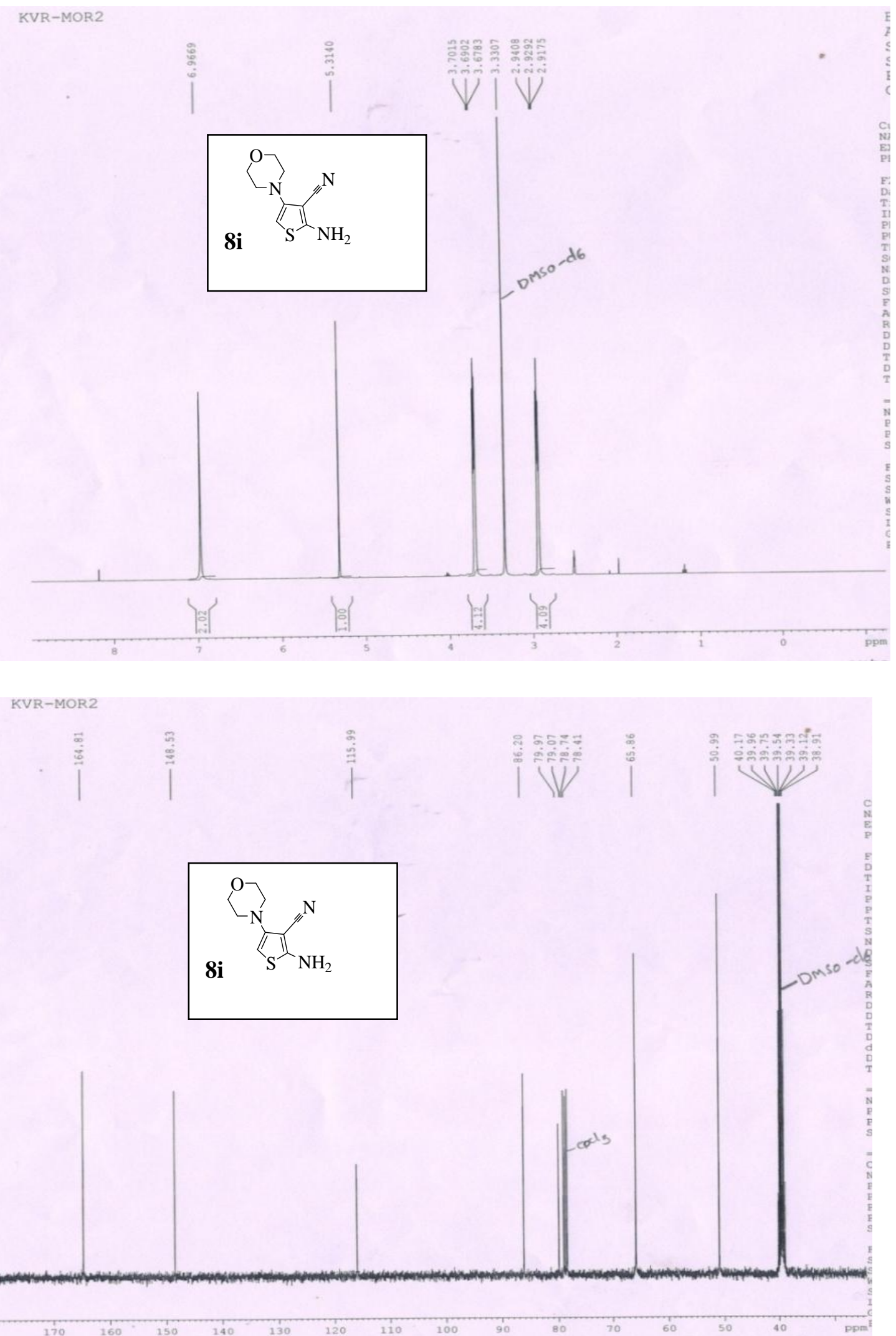


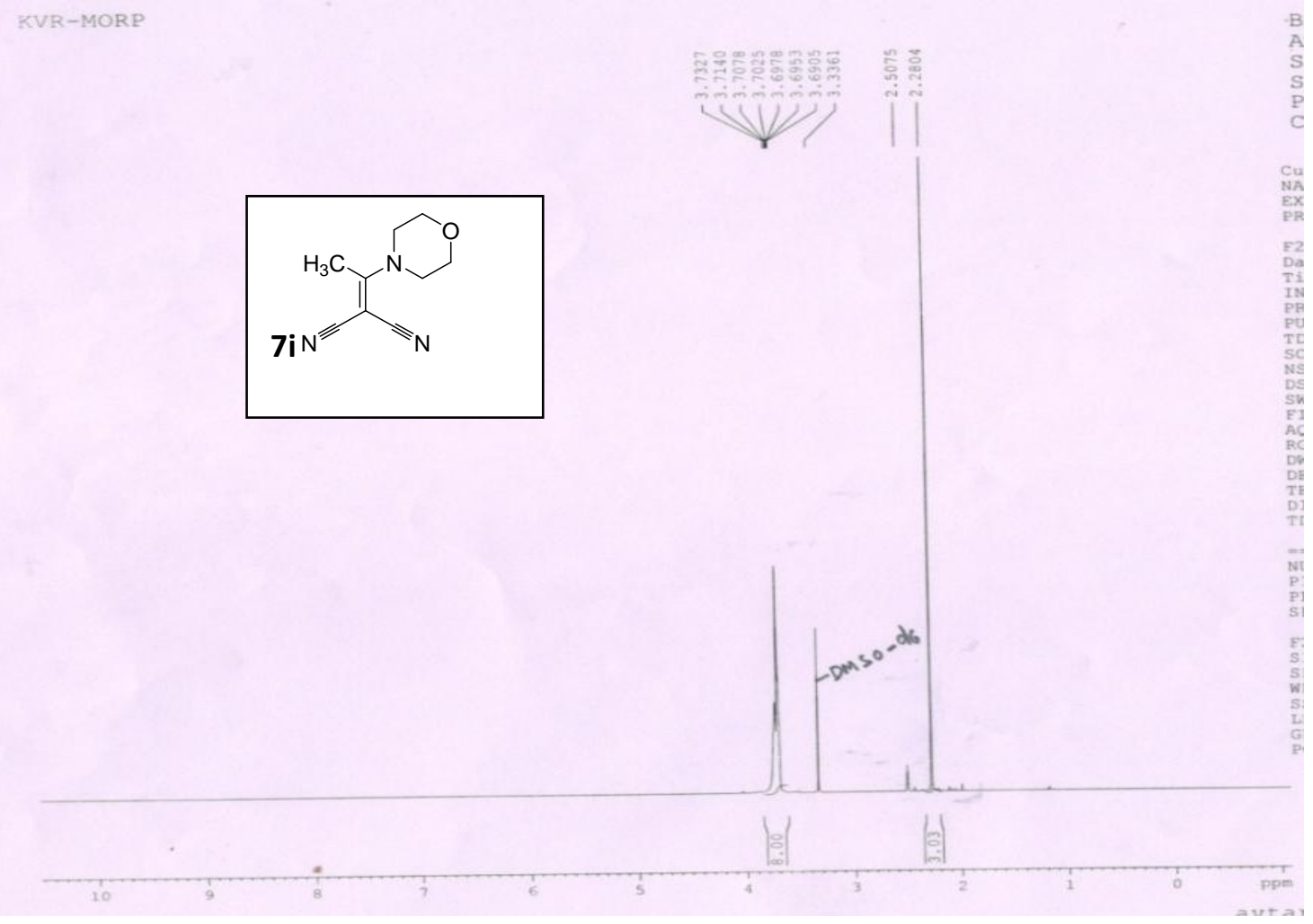

KVR-MORE
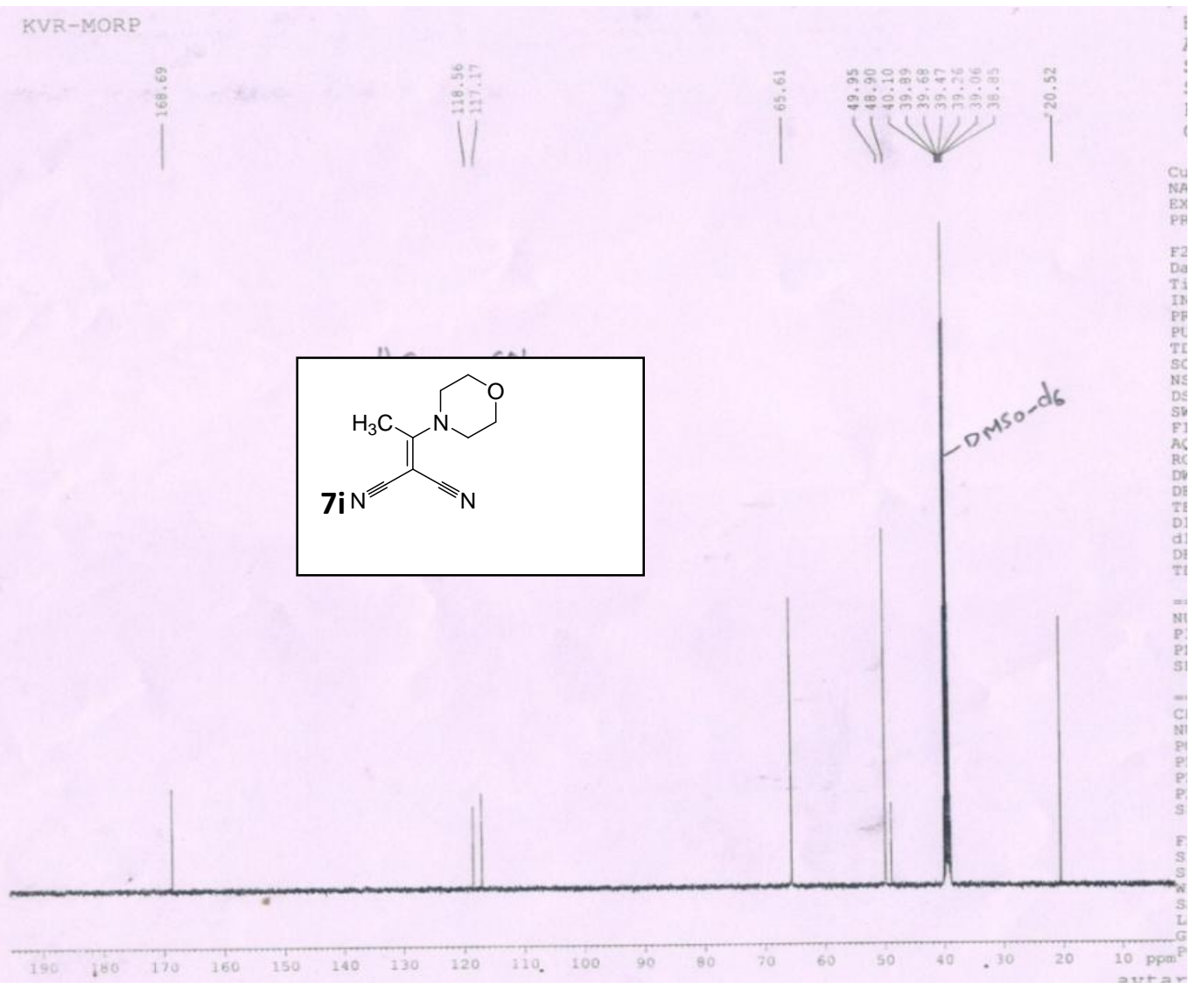